\title{
Nutritional risk factors are associated with postoperative complications after pancreaticoduodenectomy
}

\author{
Jong Hun Kim, Huisong Lee, Hyun Hwa Choi, Seog Ki Min, Hyeon Kook Lee \\ Division of HBP surgery, Department of Surgery, Ewha Womans University Mokdong Hospital, Ewha Womans University School of \\ Medicine, Seoul, Korea
}

\begin{abstract}
Purpose: The purpose of this study was to investigate whether the indicators of nutritional risk screening tool are associated with postoperative complications following pancreaticoduodenectomy (PD).

Methods: We investigated whether nutritional risk is associated with postoperative complications based on the medical records of 128 patients who underwent PD from 2010. The tool was composed of 6 risk factors: albumin, total lymphocyte count, body mass index, weight loss, dietary intake loss, and nutritional symptoms. The patients were divided into 2 groups: a nutritional risk group and a nonrisk group. The rates of general complications and postoperative pancreatic fistula (POPF) were investigated according to this nutritional status.

Results: There were 65 patients who did not have any risk factors. However, 63 patients had one risk factor or more. In the nonrisk group, the overall complication rate and serious complication rate were $30.8 \%$ and $15.4 \%$, respectively. If there were one or more risk factors, the overall and serious complication rates were $59.5 \%$ and $41.3 \%$, respectively $\mathrm{P}=0.001$ and $\mathrm{P}=0.001$, respectively). The rate of clinically relevant POPF (grade $\mathrm{B}$ or $\mathrm{C}$ ) was $9.2 \%$ in the nonrisk group. However, this rate was $23.8 \%$ in the NRS risk group $(P=0.029)$. In multivariate analysis, the NRS risk group was a significant factor of clinically relevant POPF lodds ratio, 9.878; 95\% confidence interval, 1.527-63.914; $\mathrm{P}=0.016$ ).

Conclusion: There were statistically significant associations between complications and nutritional indicators. A comprehensive analysis of nutritional parameters will help predict postoperative complications.

[Ann Surg Treat Res 2019;96(4):201-207]
\end{abstract}

Key Words: Pancreaticoduodenectomy, Postoperative complications, Mortality, Survival

\section{INTRODUCTION}

More than $30 \%$ of patients are undernourished, not only of hospitalized patients but also of nonhospitalized patients undergoing surgical procedures [1,2]. It has been suggested that nutritional depletion increases the risk of postoperative complications, such as surgical site infection, infectious disease, etc. [3-6]. For this reason, various screening tools have been developed and used in order to assess preoperative nutritional status. These nutritional risk screening (NRS) tools include subjective global assessment (SGA), patient-generated SGA (PGSGA), abridged patient-generated SGA (aPG-SGA), malnutrition universal screening tool (MUST), nutritional risk index (NRI), Onodera's prognostic nutritional index (PNI), and NRS-2002 [2,3,7-12].

Perioperative mortality has become a rare event following pancreaticoduodenectomy (PD), occurring in less than $5 \%$ of cases at high-volume pancreatic surgery centers [13-15]. Despite a significant reduction in mortality after PD, however, morbidity remains high, ranging from $30 \%-60 \%$ in some reports $[14,16,17]$.
Received September 17, 2018, Revised November 20, 2018, Accepted December 11, 2018

\section{Corresponding Author: Huisong Lee}

Department of Surgery, Ewha Womans University Mokdong Hospital, Ewha Womans University School of Medicine, 1071 Anyangcheon-ro, Yangcheon-gu, Seoul 07985, Korea

Tel: +82-2-2650-5694, Fax: +82-2-2644-7984

E-mail: denebhs@gmail.com

ORCID code: https://orcid.org/0000-0002-3565-6064
Copyright (C) 2019, the Korean Surgical Society

(c) Annals of Surgical Treatment and Research is an Open Access Journal. All articles are distributed under the terms of the Creative Commons Attribution NonCommercial License (http://creativecommons.org/licenses/by-nc/4.0/) which permits unrestricted non-commercial use, distribution, and reproduction in any medium, provided the original work is properly cited. 
The most serious morbidity following PD is pancreatic fistula $(\mathrm{PF})$, which can occur in $20 \%$ of patients [17-19]. Because of serious complications such as postoperative pancreatic fistula (POPF), the perioperative mortality rate of $\mathrm{PD}$ in high-volume centers is reportedly $1 \%$ to $2 \%$, and even as high as $40 \%[3,20]$. This POPF is associated with prognostic nutrition indexes such as serum albumin and total lymphocyte count $[3,21,22]$. Therefore, the nutritional index is a risk factor of postoperative complication after PD.

However, there is no nutritional screening tool widely accepted as the gold standard for detecting patients at risk of malnutrition and predicting postoperative complications. In previous reports, fragmentary and partial factors have been identified to assess the risk of malnutrition in the individual NRS tools mentioned above. Therefore, we investigated subjective parameters such as weight loss, dietary intake loss, and nutrition impact symptoms in addition to objective parameters such as albumin, total lymphocyte count, and body mass index (BMI). In this study, we have developed a model to analyze and evaluate nutritional indicators in an integrated manner. The aim of this study was to determine if the NRS tool could predict postoperative complications in patients who underwent $\mathrm{PD}$.

\section{METHODS}

This study was approved by the Institutional Review Board of Ewha Womans University Mokdong Hospital (2018-06-034). Written informed consent was waived by the IRB. Since January 2010, we have prospectively investigated nutritional risk factors such as albumin, total lymphocyte count, BMI, weight loss, dietary intake loss, and nutrition impact symptoms. Patients who underwent PD for periampullary cancer in Ewha Womans University Mokdong Hospital from January 2010 to December 2016 were identified retrospectively. As a concept of PD, the operative procedures performed included conventional Whipple operation, pylorus-preserving pancreaticoduodenectomy (PPPD), and laparoscopic PPPD. Periampullary cancer included pancreatic cancer, distal common bile duct cancer, and ampulla of Vater cancer. We excluded combined surgery and any previous cancer history, except for prostate cancer, thyroid cancer, and skin cancer except for melanoma.

NRS tools such as SGA, PG-SGA, aPG-SGA, MUST, NRI, Onodera's PNI, and NRS-2002 were analyzed (Table 1). These parameters included age, albumin, total lymphocyte count, BMI, weight ratio, weight loss, dietary intake loss, nutrition impact symptoms such as dysphagia, dysfunction, physical examination, and disease severity. First, the objective parameters were age, albumin, total lymphocyte count, BMI, and weight ratio. Age was excluded because recent reports have found acceptable complications after PD in the elderly, even in

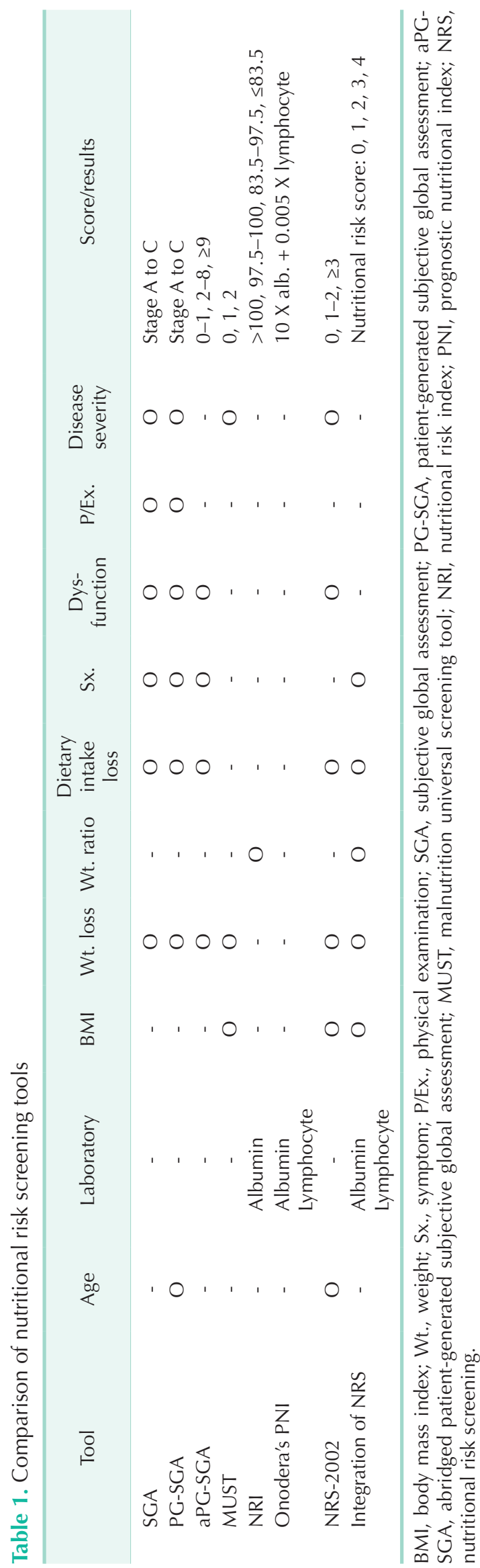


patients over 80 years of age [23-26]. Moreover, we also excluded weight ratio because it overlapped with BMI. Therefore, the objective category of NRS tools was composed of albumin, total lymphocyte count, and BMI. Second, the subjective parameters included weight loss, dietary intake loss, nutrition impact symptoms such as dysphagia, physical examination, dysfunction, and disease severity. Physical examination, dysfunction, and disease severity were excluded. Physical examination includes a subjective evaluation of three aspects of body composition: loss of subcutaneous fat (below eye, triceps, biceps, and chest), muscle wasting (clavicle, scapula, ribs, quadriceps, deltoids, etc.), and fluid states (ankle edema, sacral edema, and ascites) [3,8-10]. Dysfunction, evaluated by the patients themselves, includes working suboptimal, ambulatory, or bedridden state $[8,10]$. Disease severity, evaluated by a health professional, refers to a disease state affecting nutritional requirements. In this study, we excluded physical examination because it was performed and assessed subjectively by a health professional, e.g., physician, nurse or dietitian. Dysfunction was excluded because patients may describe it subjectively with substantial differences depending on the patient's cognitive ability and other factors. Furthermore, disease severity was also excluded because the severity of each periampullary cancer is similar, with few differences between cases. Therefore, the subjective risk factors included weight loss, dietary intake loss, and nutrition impact symptoms such as dysphagia. We defined the following variables. Definition of weight loss: If patient had a weight loss of more than $5 \%$ within the last 6 months. Dietary intake loss and nutritional symptoms were subjectively assessed by nurse or nutritionist with interview. Patients were defined as a significant decrease in the amount of food during the last 2 weeks or having complaints such as difficulty swallowing.

We analyzed 128 patients who underwent PD for periampullary cancer. The patients were divided into two groups according to the risk factors: the non-risk group $(65$ patients) and the NRS risk group (63 patients). The nonrisk group was defined as patients without NRS risk factor, and the NRS risk group was defined as patients with at least one NRS risk factor. Following this classification, patient characteristics and postoperative complications were retrospectively investigated in each group.

Patient characteristics included sex, age, BMI, weight loss, pancreatic duct size, preoperative laboratory findings such as albumin and lymphocyte, and postoperative outcomes such as total complications, serious complications, estimated blood loss (EBL), POPF, clinically relevant POPF (CR-POPF), readmission, and postoperative hospital duration. We reviewed CT images to measure pancreatic duct size at the portal vein level, and confirmed the EBL in surgical records and anesthesia records. Statistical analysis was also performed to analyze the risk factors of POPF. However, the textures of pancreas were not recorded during surgery, so it could not be analyzed for prediction of POPF.

Postoperative complications included general complications and complications specific to pancreatic surgery. General complications included wound dehiscence, complicated fluid collection, ileus, delayed gastric emptying, biliary leakage, incisional hernia, pleural effusion, pneumonia, voiding difficulty, glucose intolerance, portal vein thrombosis, sepsis, colitis, and acute cerebral infarction. Complication severity was classified according to Clavien-Dindo classification grade [27]. Clavien-Dindo classification grade II or more was considered significant. In addition, the specific complication of pancreatic surgery included only POPF after PD. The diagnosis of POPF was made with the drain output of any measurable volume of fluid on or after postoperative day 3 with amylase content 3 times greater than serum amylase activity, according to the International Study Group for Pancreatic Fistula (ISGPF) [21,28]. Severity of POPF was graded as grade A, B, or C $[15,21,28]$. Grades B and C POPF was defined as CR-POPF. Postoperative complications were investigated for 30 days after surgery. Mortality was defined as death within 90 days following surgery.

The primary end-point of the study was overall complications. The second end-point was the postoperative recovery course such as readmission, postoperative hospital duration, and complication severity.

The categorical variables are presented as number (percent). The continuous variables are presented as mean \pm standard deviation. The categorical variables were compared with the Chi-square, Fisher exact, or Student t-test. Multivariate analysis was performed for all variables using a logistic regression test. All statistical significances were determined at $\mathrm{P}<0.05$.

\section{RESULTS}

\section{Patient characteristics}

Of the 128 study patients, 65 patients (51\%) were in the nonrisk group and 63 patients (49\%) were in the risk group. There were 39 males and 26 females with mean age $63.7 \pm$ 12.2 years in the nonrisk group, and 38 males and 25 females with mean age $68.1 \pm 12.6$ years in the risk group. There was no difference in term of gender composition between the 2 groups, however, the mean age was significantly higher in the risk group than in the nonrisk group $(68.1 \pm 12.6$ vs. $63.7 \pm$ $12.2, \mathrm{P}=0.043)$. BMI was significantly lower in the risk group than in the nonrisk group $(23.1 \pm 3.5$ vs. $24.4 \pm 3.1, \mathrm{P}=0.029)$. Moreover, preoperative laboratory findings were compared between the nonrisk group and the NRS risk group. There were also significant differences in albumin $(3.2 \pm 0.5$ vs. $3.9 \pm 0.4$, $\mathrm{P}<0.001)$ and total lymphocyte count $(1,204 \pm 509$ vs. 1,908 \pm 
447, P < 0.001), favoring the nonrisk group. Furthermore, there was significantly more weight loss in the risk group than in the nonrisk group ( $7 \%$ vs. $0 \%, P=0.006$ ). Additionally, the diameter of pancreatic duct size was significantly larger in in the risk group than in the non-risk group $(3.2 \pm 2.1$ vs. $2.5 \pm 1.4, \mathrm{P}=$ 0.042) (Table 2).

\section{Postoperative outcomes}

There were no significant differences between the 2 groups in terms of EBL, POPF, and readmission. However, CR-POPF (grade $\mathrm{B}$ or $\mathrm{C}$ ) rates were significantly higher in the NRS risk group than in the nonrisk group ( $24 \%$ vs. $9 \%, \mathrm{P}=0.029)$. Also, the risk group was associated with a higher rate of overall complications (60\% vs. 31\%, P = 0.001) and serious complications (41\% vs. $15 \%$, $\mathrm{P}=0.001$ ), as well as a longer postoperative hospital duration (22.1 \pm 12.0 vs. $18.1 \pm 7.4, \mathrm{P}=0.022$ ) (Table 3).

Of the postoperative general complications, there were no differences between the 2 groups in terms of wound dehiscence, complicated fluid collection, ileus, delayed gastric emptying, biliary leakage, incisional hernia, pleural effusion,

Table 2. Characteristics of study patients $(n=128)$

\begin{tabular}{lccr}
\hline \multicolumn{1}{c}{ Characteristic } & $\begin{array}{c}\text { Nonrisk } \\
\text { group } \\
(\mathrm{n}=65)\end{array}$ & $\begin{array}{c}\text { Risk } \\
\text { group } \\
(\mathrm{n}=63)\end{array}$ & P-value $^{\mathrm{a})}$ \\
\hline Sex, male:female & $39: 26$ & $38: 25$ & 0.971 \\
Age $(\mathrm{yr})$ & $63.7 \pm 12.2$ & $68.1 \pm 12.6$ & 0.043 \\
Body mass index $\left(\mathrm{kg} / \mathrm{m}^{2}\right)$ & $24.4 \pm 3.1$ & $23.1 \pm 3.5$ & 0.029 \\
Albumin $(\mathrm{g} / \mathrm{dL})$ & $3.9 \pm 0.4$ & $3.2 \pm 0.5$ & $<0.001$ \\
Lymphocyte $(/ \mu \mathrm{L})$ & $1,908 \pm 447$ & $1,204 \pm 509$ & $<0.001$ \\
Weight loss $(\mathrm{kg})$ & $0(0)$ & $7(11)$ & 0.006 \\
Pancreatic duct size $(\mathrm{mm})$ & $2.5 \pm 1.4$ & $3.2 \pm 2.1$ & 0.042 \\
\hline
\end{tabular}

Values are presented as number, mean \pm standard deviation, number (\%).

${ }^{a}$ Chi-square, Fisher exact test, or Student t-test. voiding difficulty, glucose intolerance, portal vein thrombosis, sepsis, colitis, and acute cerebral infarction. However, postoperative pneumonia was seen significantly more in the NRS risk group than in the nonrisk group ( $8 \%$ vs. $0 \%, P=0.027$ ).

Table 3. Postoperative complications $(n=128)$

\begin{tabular}{lccc}
\hline \multicolumn{1}{c}{ Characteristic } & $\begin{array}{c}\text { Nonrisk } \\
\text { group } \\
(\mathrm{n}=65)\end{array}$ & $\begin{array}{c}\text { Risk } \\
\text { group } \\
(\mathrm{n}=63)\end{array}$ & P-value $^{\mathrm{a})}$ \\
\hline Total complication & $20(31)$ & $38(60)$ & 0.001 \\
Serious complication & $10(15)$ & $26(41)$ & 0.001 \\
Estimated blood loss $(\mathrm{mL})$ & $643 \pm 409$ & $782 \pm 667$ & 0.155 \\
Pancreatic fistula & $42(65)$ & $36(57)$ & 0.331 \\
Pancreatic fistula & $6(9)$ & $15(24)$ & 0.029 \\
$\quad$ grade B or C) & & & \\
Wound dehiscence, & $5(8)$ & $9(14)$ & 0.232 \\
$\quad$ superficial or deep SSI & & & \\
Complicated fluid & $5(8)$ & $7(11)$ & 0.507 \\
$\quad$ collection, organ & & & \\
space SSI & $2(3)$ & $3(5)$ & 0.623 \\
Ileus & $2(3)$ & $0(0)$ & 0.496 \\
Delayed gastric emptying & $1(2)$ & $0(0)$ & 0.999 \\
Biliary leakage & $1(2)$ & $0(0)$ & 0.999 \\
Incisional hernia & $1(2)$ & $4(6)$ & 0.204 \\
Pleural effusion & $0(0)$ & $5(8)$ & 0.027 \\
Pneumonia & $0(0)$ & $2(3)$ & 0.240 \\
Voiding difficulty & $0(0)$ & $1(2)$ & 0.492 \\
Glucose intolerance & $0(0)$ & $1(2)$ & 0.492 \\
Portal vein thrombosis & $0(0)$ & $2(3)$ & 0.240 \\
Sepsis & $0(0)$ & $1(2)$ & 0.492 \\
Colitis & $0(0)$ & $1(2)$ & 0.492 \\
Acute cerebral infarction & $13(17)$ & $13(21)$ & 0.621 \\
Readmission & & & \\
Hospital duration (day) & $18.1 \pm 7.4$ & $22.1 \pm 12.0$ & 0.022 \\
\hline
\end{tabular}

Values are presented as number (\%) or mean \pm standard deviation.

SSI, surgical site infection.

${ }^{a)}$ Chi-square, Fisher exact test, or Student t-test.

Table 4. Multivariate analysis for CR-POPF

\begin{tabular}{lccccc}
\hline \multicolumn{1}{c}{ Factor } & \multicolumn{2}{c}{ Univariate analysis } & & \multicolumn{2}{c}{ Multivariate analysis } \\
\cline { 2 - 3 } & OR & P-value & OR & $95 \%$ Cl & P-value ${ }^{\text {a) }}$ \\
NRS risk group & 3.010 & 0.034 & 9.878 & $1.527-63.914$ & 0.016 \\
Female & 0.716 & 0.506 & 0.429 & $0.129-1.431$ & 0.169 \\
Age & 1.006 & 0.776 & 0.997 & $0.952-1.044$ & 0.893 \\
Body mass index & 0.999 & 0.994 & 1.082 & $0.903-1.297$ & 0.393 \\
Albumin & 0.473 & 0.111 & 0.545 & $0.151-1.964$ & 0.010 \\
Lymphocyte count (k) & 1.274 & 0.546 & 4.845 & $1.460-16.083$ & 0.034 \\
Pancreatic duct size & 0.788 & 0.185 & 0.602 & $0.376-0.963$ & 0.566 \\
Estimated blood loss & 1.000 & 0.438 & 1.000 & $0.999-1.001$ & \\
\hline \hline
\end{tabular}

CR-POPF, clinically relevant-postoperative pancreatic fistula; OR, odds ratio; Cl, confidence interval; NRS, nutritional risk screening. a) Logistic regression test. 
As a concept of complications specific to pancreatic surgery, POPF was diagnosed in 78 of 128 patients (61\%) according to the ISGPF criteria. If the severity of POPF was classified as grade $\mathrm{B}$ and C, CR-POPF (grade B or C) occurred in 21 of 128 patients (16\%). In the nonrisk group, the POPF and CR-POPF rates were $65 \%$ and $9 \%$ of 65 patients, respectively. In the NRS risk group, however, the POPF and CR-POPF rates were $57 \%$ and $24 \%$ of 63 patients, respectively $(\mathrm{P}=0.331$ and $\mathrm{P}=0.029$, respectively). Therefore, the NRS risk group was associated with a higher rate of CR-POPF (24\% vs. 9\%, P = 0.029) (Table 3). There was no 90day mortality in this series.

Univariate analysis showed a significant difference in the NRS risk group (odds ratio [OR], 3.010; $P=0.034$ ). Furthermore, multivariate analysis revealed being in the NRS risk group, a lower total lymphocyte count, and a larger pancreatic duct size to be significant risk factors for CR-POPF (NRS risk group: OR, 9.878; 95\% confidence interval [CI], 1.527-63.914; $\mathrm{P}=0.016$; lower total lymphocyte count: OR, 4.845; 95\% CI, 1.460-16.083; $\mathrm{P}=0.010$; larger pancreatic duct size: $\mathrm{OR}, 0.602 ; 95 \% \mathrm{CI}$, $0.376-0.963 ; \mathrm{P}=0.034)$. In other words, NRS risk group was a significant factor of CR-POPF (Table 4).

\section{DISCUSSION}

We have prospectively investigated preoperative nutritional status since January 2010. We retrospectively reviewed our series of 128 patients to analyze whether nutritional risk factors are associated with postoperative complications. Of the 128 patients, 65 patients (51\%) had no NRS risk, and 63 patients $(49 \%)$ had at least one NRS risk factor according to the integration of NRS tools. Among the preoperative variables, BMI and laboratory findings such as albumin and total lymphocyte count were significantly lower in the NRS risk group than in the nonrisk group, while age was significantly higher in the NRS risk group. There was significantly more weight loss in the NRS risk group than in the nonrisk group. In addition, NRS risk group was associated with a larger pancreatic duct diameter size. Regarding the postoperative variables, NRS risk group was associated with a higher rate of total complications, serious complications, CR-POPF (grade B or C), and a longer postoperative hospital duration. In other words, CR-POPF, the only specific complication following PD, was seen significantly more in the NRS risk group ( $24 \%$ vs. $9 \%, P=0.029$ ). Of the individual general complications, only pneumonia was seen significantly more in the NRS risk group ( $8 \%$ vs. $0 \%, \mathrm{P}=0.027)$. As a result, postoperative complication and CR-POPF rates were significantly higher in the NRS risk group with one or more nutritional risks as identified by the objective factors of NRS tool. As analyzed in Table 4, being in the NRS risk group was identified as a statistically significant and independent predictor of $\mathrm{CR}-\mathrm{POPF}$ after $\mathrm{PD}$ in multivariate analysis ( $\mathrm{P}=$
$0.016)$ as well as univariate analysis ( $\mathrm{P}=0.034)$. Therefore, these findings suggest that the integration of NRS system can be a preoperative predictive model for postoperative complications and CR-POPF in patients undergoing PD.

The reported NRS tools for predicting postoperative complications include SGA, PG-SGA, aPG-SGA, MUST, NRI, Onodera's PNI, and NRS-2002 [2,3,7-12]. Although these various NRS tools have already been recognized in preoperative risk stratification [3], NRS scoring systems collectively integrating objective parameters (albumin, total lymphocyte count, and BMI) and subjective parameters (weight loss, dietary intake loss, and nutrition impact symptoms) have not been employed in any previous studies. Of the previous NRS tools, aPG-SGA, MUST, and NRS-2002 belonged to the NRS scoring system, however, objective parameters such as albumin, total lymphocyte count, and/or BMI were excluded. In a recent investigation, scoring systems combing several factors have been proposed to more accurately predict the risk of POPF [19,21]. In this study, thus, we have integrated and scored the objective and subjective parameters together to comprehensively assess preoperative nutritional status. Finally, among the various parameters, the nutritional risk tool included albumin, total lymphocyte count, BMI, and subjective risk factors (weight loss, dietary intake loss, and dysphagia). In this study, we found out the NRS risk group was shown to be independently associated with CR-POPF after PD by multivariate analysis.

The incidence of CR-POPF (grade B or C) was 16\%, which was similar to that reported by previous studies $(13.2 \%-17 \%)$ $[16,21]$. In the nonrisk group, the CR-POPF rate was $9 \%$. If there was at least one NRS risk factor, the rate rose to $24 \%$ $(P=0.029)$. The reason why being in the NRS risk group was associated with a higher rate of CR-POPF seems to be that physical tolerance against surgical trauma becomes weaker with nutritional depletion. The other reason why is that nutrition plays an integral role in PD for periampullary cancer, not only preoperatively but also postoperatively [3]. For these reasons, if patients are identified as part of the NRS risk group prior to surgery, nutritional intervention should be provided early in treatment so as to prevent CR-POPF after PD. This preoperative nutritional support may be very important in the postoperative recovery course, because CR-POPF is the principal cause of postoperative morbidity and mortality [20,29].

Furthermore, postoperative pneumonia was significantly more in the NRS risk group; the reason for this may be that the undernourished patients are in poor physical condition, causing their immune systems to weaken. This finding may be associated with the lower lymphocyte count in the NRS risk group found by multivariate analysis (OR, $4.845 ; 95 \% \mathrm{CI}$, 1.460-16.083; $\mathrm{P}=0.010$ ). To prevent postoperative pneumonia, when we detect patients at risk of malnutrition, we should also support preoperative nutrition. Moreover, the result that the CR- 
POPF and pneumonia rates were higher in the NRS risk group may be associated with a longer postoperative hospital duration (22.1 \pm 12.0 vs. $18.1 \pm 7.4, \mathrm{P}=0.022)$. If patients are screened for NRS nutritional risk, and are appropriately supported with preoperative nutrition, then the average mortality and postoperative hospital duration as well as overall complications can be expected to decrease in the future. In future studies, the efficacy of preoperative nutritional support in patients in the NRS risk group should be prospectively evaluated.

The present study has several limitations. First, the main limitations of this study are its retrospective nature as well as selection bias. The pancreas texture, known to be associated with POPF, could not be analyzed due to the limitation of the retrospective study. Second, the possibility of bias related to the historical background cannot be eliminated. For example, it is not possible to eliminate the possibility of bias related to subjective risk factors such as weight loss, dietary intake loss, and nutrition impact symptoms such as dysphagia. In addition to these biases, patients were overestimated because a subjective risk factor is scored as one point in analysis, even if there is one of weight loss, dietary intake loss, and dysphagia.
In fact, the NRS risk group was measured at $49 \%$ in our study, unlike the more than $30 \%$ rate seen in previous studies. Third, the number of patients is relatively small and may be underpowered for some statistical analyses. To further validate our present findings, a prospective study in a larger number of patients is needed.

In conclusion, there were statistically significant associations between complications and nutritional indicators. A comprehensive analysis of nutritional parameters will help predict postoperative complications and CR-POPF after PD.

\section{CONFLICTS OF INTEREST}

No potential conflict of interest relevant to this article was reported.

\section{ACKNOWLEDGEMENTS}

This work was supported by the Ewha Womans University Research Grant of 2017.

\section{REFERENCES}

1. McWhirter JP, Pennington CR. Incidence and recognition of malnutrition in hospital. BMJ 1994:308:945-8.

2. Velasco C, Garcia E, Rodriguez V, Frias L, Garriga R, Alvarez J, et al. Comparison of four nutritional screening tools to detect nutritional risk in hospitalized patients: a multicentre study. Eur J Clin Nutr 2011;65: 269-74.

3. Afaneh C, Gerszberg D, Slattery E, Seres DS, Chabot JA, Kluger MD. Pancreatic cancer surgery and nutrition management: a review of the current literature. Hepatobiliary Surg Nutr 2015:4:59-71.

4. La Torre M, Ziparo V, Nigri G, Cavallini M, Balducci G, Ramacciato G. Malnutrition and pancreatic surgery: prevalence and outcomes. J Surg Oncol 2013;107:702-8.

5. Ahmad SA, Edwards MJ, Sutton JM, Grewal SS, Hanseman DJ, Maithel SK, et al. Factors influencing readmission after pancreaticoduodenectomy: a multi-institutional study of 1302 patients. Ann Surg 2012;256:529-37.
6. Zhou W, Xu X, Yan J, Mou Y. Nutritional risk is still a clinical predictor of postoperative outcomes in laparoscopic abdominal surgery. Surg Endosc 2013;27:256974.

7. White JV, Guenter P, Jensen G, Malone A, Schofield M; Academy of Nutrition and Dietetics Malnutrition Work Group, et al. Consensus statement of the Academy of Nutrition and Dietetics/American Society for Parenteral and Enteral Nutrition: characteristics recommended for the identification and documentation of adult malnutrition (undernutrition). J Acad Nutr Diet 2012;112:730-8.

8. Ferguson M. Patient-generated subjective global assessment. Oncology (Williston Park) 2003:17(2 Suppl 2):13-4.

9. Detsky AS, McLaughlin JR, Baker JP, Johnston N, Whittaker S, Mendelson RA, et al. What is subjective global assessment of nutritional status? JPEN J Parenter Enteral Nutr 1987;11:8-13.

10. Bauer J, Capra S, Ferguson M. Use of the scored Patient-Generated Subjective Global Assessment (PG-SGA) as a nutrition assessment tool in patients with cancer. Eur J Clin Nutr 2002:56:779-85.

11. Ottery FD. Definition of standardized nutritional assessment and interventional pathways in oncology. Nutrition 1996;12(1 Suppl):S15-9.

12. Kondrup J, Allison SP, Elia M, Vellas B, Plauth M; Educational and Clinical Practice Committee, et al. ESPEN guidelines for nutrition screening 2002. Clin Nutr 2003;22:415-21.

13. Cameron JL, Riall TS, Coleman J, Belcher KA. One thousand consecutive pancreaticoduodenectomies. Ann Surg 2006;244: 10-5.

14. Ansorge C, Lindstrom P, Strommer L, Blomberg J, Lundell L, Andren-Sandberg A, et al. Assessing surgical quality: comparison of general and procedure-specific morbidity estimation models for the risk adjustment of pancreaticoduodenectomy outcomes. World J Surg 2014:38:2412-21. 
15. Hackert T, Hinz U, Pausch T, Fesenbeck I, Strobel O, Schneider L, et al. Postoperative pancreatic fistula: we need to redefine grades B and C. Surgery 2016;159:872-7.

16. Kimura W, Miyata H, Gotoh M, Hirai I, Kenjo A, Kitagawa Y, et al. A pancreaticoduodenectomy risk model derived from 8575 cases from a national single-race population (Japanese) using a web-based data entry system: the 30-day and inhospital mortality rates for pancreaticoduodenectomy. Ann Surg 2014;259:77380.

17. Schmidt CM, Turrini O, Parikh P, House MG, Zyromski NJ, Nakeeb A, et al. Effect of hospital volume, surgeon experience, and surgeon volume on patient outcomes after pancreaticoduodenectomy: a singleinstitution experience. Arch Surg 2010; 145:634-40.

18. Greenblatt DY, Kelly KJ, Rajamanickam V, Wan Y, Hanson T, Rettammel R, et al. Preoperative factors predict perioperative morbidity and mortality after pancreaticoduodenectomy. Ann Surg Oncol 2011;18: 2126-35.

19. Roberts KJ, Sutcliffe RP, Marudanayagam $\mathrm{R}$, Hodson J, Isaac J, Muiesan P, et al.
Scoring system to predict pancreatic fistula after pancreaticoduodenectomy: a UK multicenter study. Ann Surg 2015;261: 1191-7.

20. Muscari F, Suc B, Kirzin S, Hay JM, Fourtanier G, Fingerhut A, et al. Risk factors for mortality and intra-abdominal complications after pancreatoduodenectomy: multivariate analysis in 300 patients. Surgery 2006;139:591-8.

21. Sato N, Tamura T, Minagawa N, Hirata $\mathrm{K}$. Preoperative body mass index-to-prognostic nutritional index ratio predicts pancreatic fistula after pancreaticoduodenectomy. Hepatobiliary Surg Nutr 2016;5: 256-62.

22. Sierzega M, Niekowal B, Kulig J, Popiela T. Nutritional status affects the rate of pancreatic fistula after distal pancreatectomy: a multivariate analysis of 132 patients. J Am Coll Surg 2007;205:52-9.

23. Melis M, Marcon F, Masi A, Pinna A, Sarpel U, Miller G, et al. The safety of a pancreaticoduodenectomy in patients older than 80 years: risk vs. benefits. HPB (Oxford) 2012;14:583-8.

24. Tani M, Kawai M, Hirono S, Ina S, Miyazawa M, Nishioka R, et al. A pancrea- ticoduodenectomy is acceptable for periampullary tumors in the elderly, even in patients over 80 years of age. J Hepatobiliary Pancreat Surg 2009;16:675-80.

25. Lee MK, Dinorcia J, Reavey PL, Holden MM, Genkinger JM, Lee JA, et al. Pancreaticoduodenectomy can be performed safely in patients aged 80 years and older. J Gastrointest Surg 2010;14:1838-46.

26. Kim JH, Min SK, Lee H, Hong G, Lee HK. The safety and risk factors of major hepatobiliary pancreatic surgery in patients older than 80 years. Ann Surg Treat Res 2016;91:288-94.

27. Dindo D, Demartines N, Clavien PA. Classification of surgical complications: a new proposal with evaluation in a cohort of 6336 patients and results of a survey. Ann Surg 2004;240:205-13.

28. Bassi C, Dervenis C, Butturini G, Fingerhut A, Yeo C, Izbicki J, et al. Postoperative pancreatic fistula: an international study group (ISGPF) definition. Surgery 2005; 138:8-13.

29. Martin RC 2nd, Brennan MF, Jaques DP. Quality of complication reporting in the surgical literature. Ann Surg 2002;235:80313 